\title{
Nano-Forensic: New Perspective and Extensive Applications in Solving Crimes
}

\author{
Sonali Kesarwani $^{1}$ (D), Kapil Parihar ${ }^{1}$ (D), Mahipal Singh Sankhla ${ }^{1, *}$ (D), Rajeev Kumar ${ }^{2}$ (D) \\ 1 Research Scholars, Department of Forensic Science, School of Basic and Applied Sciences, Galgotias University, \\ Greater Noida, India \\ Associate Professor Department of Forensic Science, School of Basic and Applied Sciences, Galgotias University, \\ Greater Noida, India \\ * Correspondence: mahipal4n6@gmail.com;
}

Received: 4.09.2020; Revised: 27.09.2020; Accepted: 29.09.2020; Published: 2.10.2020

\begin{abstract}
Nanotechnology has continued to prove its dominance with vast applications to traditional methods in medical, electronic, engineering, and other fields. In forensic science, nanotechnology research has provided a new perspective for real-time crime investigation and developed advanced nanosensors, nano-manipulators, and nano-imaging tools for visualization. Often, nanotechnology aids in enhancing and improving the efficiency of already existing and applied forensic techniques with high accuracy, sensitivity, and reducing the time required. So, this paper reviews the vital applications of classic tools of nanotechnology for examining questioned documents, age of bloodstains, and time since death, along with its application in improving PCR efficiency, DNA analysis, and explosive detection. It also attempts to highlight the use of advanced nanotechnology instruments for illicit drug screening. Nano-based techniques hold an immense future in fingerprinting and security features. Therefore, this paper also offers insights into the applications of nanoparticles in detecting latent fingerprints.
\end{abstract}

Keywords: nano-forensics; nanotechnology; nanoparticles; crime scene investigation; application.

(C) 2020 by the authors. This article is an open-access article distributed under the terms and conditions of the Creative Commons Attribution (CC BY) license (https://creativecommons.org/licenses/by/4.0/).

\section{Introduction}

The emergence of nanotechnology allows the changes and control of atoms and molecules of a matter at their atomic and molecular level to form some novel materials and devices with more diverse and potentially extraordinary properties. It is a fast expanding research area with vast applications in different fields. The word 'Nano' means dwarf or small, which refers to one billionth size, i.e., $10^{-9}$, and equals to about one nanometer $(\mathrm{nm})$. In other words, it is about 3-5 atoms wide or 40,000 times lesser than the thickness of human hair or a virus $(100 \mathrm{~nm})$ [1]. Thus, nanotechnology deals with the emerging materials of size ranging from 1 to $100 \mathrm{~nm}$ [2]. Due to its vast applicability in various fields, nanotechnology has also made its place in forensic science, i.e., nano-forensics. Nano-forensics is the latest and fastgrowing field of innovation that deals with the application of nano-based technology. The evolution of nanosensors replaces the large bulky instruments into a much smaller chip-based platform. This greatly aids in crime investigation and also to identify anonymous evidence in a shorter analysis method of investigation with higher accuracy and sensitivity for solving crimes. Some of the upgraded analysis instruments include Atomic Force Microscopy (AFM), Scanning Probe Microscope (SPM), High-Performance Liquid Chromatography (HPLC), X- 
ray Photoelectron Spectroscopy (XPS), Differential Scanning Colorimetry (modulated DSC), FT-IR radiation, Raman-IR radiation, and Time-of-flight Mass Spectroscopy (Tof-MS) [3].

These techniques aid forensic scientists in two ways:

1. By detecting and analyzing nano-scaled samples that could not have been possible earlier because of the detection limits of previous instruments [4].

2. The novel properties of nanomaterial help in the detection and collection of critical evidence that can't be acquired previously. DNA extractions from fingerprint or palm print, heavy metals, gunshot residues, explosives are some novel approaches that ease the way of providing conclusive evidence in a court of law. There are ample applications of such technology in the nano-forensic field. Therefore, in this paper, an attempt has been made to highlight the new perspective or idea of nanotechnology with its applications in various branches of forensic science, i.e., nano-forensics [4].

\section{Applications of nanotechnology in forensic science}

Following are the various disciplines of forensic science which involves the application of the modern approach of nanotechnology:

\subsection{Nanotechnology in a questioned document.}

Examination of questioned documents (QD) has also paced technology to study the material in nano-scale (nm) [1]. In the case of forged documents where ink is involved, analysis of minute details of ink can provide several hints. This can be done by one such advanced instrument, AFM. It is a type of microscope that is capable of visualizing ink crossing by scanning the document to determine the sequence of ink strokes. Also, it provides 3-D surface morphology, which helps to determine crucial information regarding the sequence of ball pen strokes or ribbon dye [5]. Moreover, nanostructured materials based on metals, carbon, and silicon have been developed to aid Mass spectroscopy in Surface Assisted Laser Desorption/Ionization Mass Spectroscopy (SALDI-MS). Researchers have worked on solventfree gold nanoparticle assisted LDI-MS technique to detect image inks and visible dyes on banknotes and questioned documents to analyze the sequence of different ink strokes for tracing forgery or alterations [6].

\subsection{Nanotechnology in toxicology for screening drug-facilitated crime.}

In the present scenario, the modern application of nanotechnology is effectively used in forensic toxicology for the detection of various toxic materials and its quantification from numerous forensic evidence such as blood, hair, saliva, urine, vitreous humor, and even from fingerprints and skeletal remains. Detection of a wide variety of both legal medicine like paracetamol and illicit drugs like cocaine, heroin, and other barbiturates uses advanced nanotechniques like HPLC, XPS, and Tof-MS. Moreover, the advancement makes use of capillary electrophoresis, nano-gold (Au-NPs), nano-silver, and nano-titanium dioxide $\left(\mathrm{TiO}_{2}\right)$ particles coupled with SEM, TEM, and FTIR [7]. Functionalized magnetic particles with antibodies were used for the detection of various drugs and their metabolites such as morphine (a metabolite of heroin), benzoylecgonine (a metabolite of cocaine), and others from latent fingerprint [8]. Similarly, [9] reported that a microfluid system using silver nanoparticles could detect illicit drugs in saliva. Researchers have also come up with a rapid method for drug detection in human urine samples by using Dynamic-surface enhanced Raman spectroscopy 
(D-SERS) and gold nanorods (GNRs) along with the classification algorithm known as support vector machine (SVM) [10]. According to a recent study, the use of techniques like direct analysis at real-time (DART) Tof-MS and micro-extraction by packed sorbent (MEPS) and has improved the speed of drug detection by less than a minute and extraction by less than two minutes from a urine sample. Recently, a "smart" system was developed to rapidly detect and ascertain the quantity of codeine sulfate using citrate-stabilized Au-NPs as a probe and a "smartphone camera" as an analysis device. Due to the presence or absence of codeine sulfate, the camera of the smartphone was capable of detecting extremely sensitive changes in color, i.e., ultrasensitive colorimetric changes in the nanoprobe. This system of nanotechnology provided rapid, portable, and on-spot detection methods of illicit drugs [11].

\subsection{Nanotechnology in explosive detection.}

Explosive based terrorism is a majorly grown area of crime because of the easy availability of explosive-based weapons. They are simple to construct, easy to deploy, and can cause huge damage. Due to this reason, the current scenario requires sensitive and accurate explosive detection techniques. The major challenges involved in their detection are due to its vast variety, a vast number of deployment, and lack of inexpensive and sensitive sensors. Primarily, the detection process involves the collection of trace elements as vapor or particulate matter and then their analysis with a sensitive sensor system. The old techniques of detection involve a tedious process. Therefore, the requirement of technique having high sensitivity and selectivity combined with a low cost of sensors and low limit of detection (LOD) is the biggest challenge [3]. Hence, modern research and development have led to a novel nano arena of technology involving nanostructured sensors with high sensitivity, specificity, and fast detection and regeneration time of various chemical, biological as well as explosives compounds. For example, the use of polymer particles and nanoparticles along with various nanosensor devices like nano-curcumin based probes, electronic noses, nano-wires or nanotubes, and lasing plasmon nanocavity, when adhering to explosives, causes a change in one of their measurable properties with high specificity detection. Researchers have reported the highly cost-effective way of detecting trace Trinitrotoluene (TNT) by the use of curcumin as nano curcumin [12]. Also, SERS has been an ideal choice for explosive detection due to its non-destructive nature and large Raman enhancement factors [3].

\subsection{Nanotechnology in the estimation of time since death.}

One of the most crucial issues in forensic medicine and law is the estimation of time since death (TSD) or time of death. The various physical and chemical postmortem changes in a body play a vital role in providing approximate TSD. Moreover, observable changes in some of the body fluids like blood, spinal fluid, pericardial fluid, aqueous and vitreous humor, synovial fluid are also useful procedures to estimate TSD soon after death [13]. Out of these, vitreous humor $(\mathrm{VH})$ is the fluid that remains the same and does not change even after a long duration of death. This means that biochemical changes in $\mathrm{VH}$, which result in a change in the level of the amino acid, are slow. Hence, by analyzing these biochemical changes, estimation of TSD can be done precisely [14]. Recent technology has developed a smart, sensitive, and cost-effective lab-on-chip method to easily determine cysteine (amino acid), which may lead to an estimate TSD up to $96 \mathrm{hrs}$ till the concentration of cysteine in VH increases significantly and show a linear correlation with the passage of TSD [15]. Furthermore, TSD was also 
estimated by a flow cytometry-based method that measures the DNA degradation rate of the spleen and brain tissue up to $96 \mathrm{hrs}$. In the near future, fluorescent nanoparticles can help in measuring the concentration of $\mathrm{VH}$ along with its quantification by flow cytometry, which would be an advanced approach to estimate TSD more precisely [16].

\subsection{Nanotechnology in bloodstains age estimation.}

Forensic investigation of a crime scene involves a lot of reliable methods for the identification of bloodstains. However, the bloodstains age estimation remains an unsolved issue in the routine analysis [17]. So, here comes a challenge to estimate the age of bloodstains with certainty. Morphological and cellular changes of blood cells can be useful for quantitative assessment of the age of dry bloodstains. Recent studies have reported the application of AFM to resolve this one of the crucial issues of forensic science. Since AFM is a new potential tool for estimating the age of bloodstains and time of death, so potentially it is providing useful information to legal, medical experts in solving crimes. Therefore, AFM tests the changes in morphology and surface elasticity of RBC by recording force-distance curves. The pattern of elasticity decreases with time, which is more likely due to the coagulation and degradation process of bloodstains. Hence, by calibrating the elasticity curve concerning time, the bloodstains age can be estimated, which can further aid in a criminal investigation [18].

\subsection{Nanotechnology in DNA analysis: enhancement of PCR efficiency.}

The pace of heinous crime is increasing exponentially in our society. To tackle such crime, nanotechnology has a vital role. One of the most crucial corroborative evidence is the genetic material DNA, which acts as an indication or sometimes even confirms the presence or absence of a person at the crime scene. Researchers have reported the use of magnetic nanoparticles to extract the DNA from various biological samples (hair, blood, saliva, skin, semen) [19]. More advanced nano-based tools have also been developed that can read the DNA sequence in a molecule directly. Also, a DNA sequence can be analyzed by AFM by placing DNA molecules in carbon nanotubes [20]. Moreover, it has also been found that nanotechnology, i.e., Au-NPs, can be used to dramatically enhance the polymerase chain reaction (PCR) efficiency. By just adding $0.7 \mathrm{~nm}$ of $13 \mathrm{~nm}$ Au-NPs into the PCR reagent, marked improvement in the PCR efficiency was observed, i.e., decrease in reaction time while an increase in the rate of heating/cooling of thermal cycler [18]. Au-NPs have also been found to enhance electro-chemiluminescence (ECL) combined with isothermal reaction of polymerase and nicking endonuclease. Hence, the use of Au-NPs enhances sensitivity and selectivity as compared to both conventional and real-time PCR [21].

\subsection{Nanotechnology in fingerprint development and identification.}

One of the most widely found evidence in nearly every crime scene is- fingerprints. These are the most crucial elements of any crime scene as they link a crime to a suspect, so they are generalized proof of human identity. The emergence of nanotechnology techniques by the use of NPs has shown great potential in the development and identification of fingerprint to the next generation level, known as nano-fingerprints. Recent studies have revealed the application of micro X-ray fluorescence method to visualize latent fingerprints [22]. This method is contrary to the conventional chemical methods (iodine fuming, silver nitrate, ninhydrin, and cyanoacrylate). Despite several advantages of this method, it still required 
instruments and trainers to conduct such an analysis. Therefore, the current nano-based technology can be of much help to develop and analyze the fingerprint evidence on the spot with the lowest chances of error. Nowadays, different types of nanopowders are used to apply on different surfaces to visualize the latent fingerprints. Recently, researchers have synthesized novel $\mathrm{ZnO}-\mathrm{SiO}_{2}$ nanopowder, which was used along with a small particle reagent (SPR) method to develop latent fingerprint [23]. The method showed excellent results with a high level of potential finger ridge details than other conventional powder methods. Moreover, Titanium dioxide $\left(\mathrm{TiO}_{2}\right.$-NPs) powder was reported to develop fingerprint on the adhesive side of black tape and also on bloody prints [24-26]. Thus, the use of NPs like Au-NPs, ZnS (Zinc sulfide), $\mathrm{CdS}$ (cadmium sulfide), $\mathrm{CdSe}$, iron oxide, $\mathrm{TiO}_{2}$, europium oxide, molybdenum disulfide, and nobel metals nanocrystals have been reported to mark significant enhancing and developing latent fingerprints methods on various porous and non-porous surfaces with improved sensitivity, selectivity, and contrast with the background [3, 27-29].

\subsection{Nanotechnology for security tags in documents.}

The security of valuable assets and confidential documents has always been a major concern for a country. There are numerous ideas for making documents secure and counterfeitproof. For example, in passports, credit cards, or banknotes, various security features that are embedded include watermarks, security fibers, fluorescent inks, optical variable inks, holograms, and planchettes. In general practice, inorganic and organic luminescent powders like phosphors and fluorophors are used to invisibly tag a document. Nowadays, nanoparticles are being used as security features, particularly luminescent NPs such as quantum dots (Q-dots) or nano-sized luminescent phosphors and up-converters. These are being used by introducing them in inks or paper to make the document more secure. The advancement has paced up in developing various kinds of luminescent NPs that aids in formulating anti-counterfeiting inks. Therefore, the current nano-formulations are replacing the use of old fluorescent powders and dyes and providing more precise and upgraded security features [2].

\subsection{Discussion.}

In this review paper, the wide applications of nanotechnology in the discipline of forensic science, i.e., nano-forensics, have been discussed. This novel and advanced approach of nano-based materials and instruments like AFM, SALDI, D-SERS, HPLC, SEM, TEM, nanosensors, and others have been able to solve most of the crucial issues of forensic investigation, such as explosive detection, bloodstain age estimation, in QD, in the estimation of time since death, etc., hence aided in solving crimes. Application of Au-NPs has contributed to most of the branches like DNA analysis, latent fingerprint identification, drug screening. Also, the use of Q-dots as luminescent in security features, $\mathrm{CdS}, \mathrm{FeO}, \mathrm{TiO}_{2}, \mathrm{ZnS}$ nanoparticles, etc. in fingerprint detection has provided improved and more sensitive results.

\section{Conclusions}

It is concluded that the vast applications of nanoparticles, nano-based techniques, and devices in the field of forensic science have come up with fast, more accurate, sensitive, selective, and efficient methods of crime scene investigations as well as forensic evidence 
examination, which ultimately leads to solving crime. Therefore, nanotechnology, not only is a major scope in other fields, but also likely to play a major role in the coming future of forensic science as well with more advanced and sensitive ways of investigation.

\section{Funding}

This review received no external funding.

\section{Acknowledgments}

This review has no acknowledgment.

\section{Conflicts of Interest}

The author declares no conflict of interest.

\section{References}

1. Lodha, A.S.; Pandya, A.; Shukla, R.K. Nanotechnology: an applied and robust approach for forensic investigation. Forensic Res Criminol Int J 2016, 2, 00044, https://doi.org/10.15406/frcij.2016.02.00044.

2. Prasad, V.; Lukose, S.; Prasad, L. Emerging forensic applications of nanotechnology. Int J Eng Allied Sci 2016, $2,1-8$.

3. Jagriti; Jaiswal, A.K.; Jaiswal, A.K. Nanotechnology in forensic Science: Scope and Challenges,Int J Med Lab Res 2017; 2, 77-93.

4. Nanotechnology and Materials Engineering, $32^{\text {nd }}$ European Congress. Available online: https://nanotechnology-materialscience.materialsconferences.com/events-list/forensicnanotechnology(accessed on 11/06/2020).

5. Prasad, V.; Lukose, S.; Agarwal, P.; Prasad, L. Role of Nanomaterials for Forensic Investigation and Latent Fingerprinting—A Review. J. Forensic Sci. 2020, 65, 26-36, https://doi.org/10.1111/1556-4029.14172.

6. Han, Z.; Liu, H.; Wang, B.; Weng, S.; Yang, L.; Liu, J. Three-dimensional surface-enhanced Raman scattering hotspots in spherical colloidal superstructure for identification and detection of drugs in human urine. Anal. Chem. 2015, 87, 4821-4828, https://doi.org/10.1021/acs.analchem.5b00176.

7. Chen, X.; Tang, Y.; Wang, S.; Song, Y.; Tang, F.; Wu, X. Field-amplified sample injection in capillary electrophoresis with amperometric detection for the ultratrace analysis of diastereomeric ephedrine alkaloids. Electrophoresis 2015, 36, 1953-1961, https://doi.org/10.1002/elps.201500024.

8. Hazarika, P.; Jickells, S.M.; Wolff, K.; Russell, D.A. Imaging of latent fingerprints through the detection of drugs and metabolites. Angew. Chem. Int. Ed. 2008, 47, 10167-10170,

https://doi.org/10.1002/anie.200804348.

9. Andreou, C.; Hoonejani, M.R.; Barmi, M.R.; Moskovits, M.; Meinhart, C.D. Rapid Detection of Drugs of Abuse in Saliva Using Surface Enhanced Raman Spectroscopy and Microfluidics. ACS Nano 2013, 7, 7157 7164, https://doi.org/10.1021/nn402563f.

10. Dong, R.; Weng, S.; Yang, L.; Liu, J. Detection and Direct Readout of Drugs in Human Urine Using Dynamic Surface-Enhanced Raman Spectroscopy and Support Vector Machines. Anal. Chem. 2015, 87, 2937-2944, https://doi.org/10.1021/acs.analchem.5b00137.

11. Lodha, A.; Pandya, A.; Sutariya, P.G.; Menon, S.K. A smart and rapid colorimetric method for the detection of codeine sulphate, using unmodified gold nanoprobe. RSC Advances 2014, 4, 50443-50448, https://doi.org/10.1039/c4ra06269h.

12. Pandya, A.; Goswami, H.; Lodha, A.; Menon, S.K. A novel nanoaggregation detection technique of TNT using selective and ultrasensitive nanocurcumin as a probe. Analyst 2012, 137, 1771-1774, https://doi.org/10.1039/C2AN35131E.

13. Garg, V.; Oberoi, S.S.; Gorea, R.K.; Kaur, K. Changes in the levels of vitreous potassium with increasing time since death. Journal of Indian Academy of Forensic Medicine 2004, 26, 136-139.

14. Swann, L.M.; Forbes, S.L.; Lewis, S.W. Analytical separations of mammalian decomposition products for forensic science: A review. Anal. Chim. Acta 2010, 682, 9-22, https://doi.org/10.1016/j.aca.2010.09.052. 
15. Ansari, N.; Lodha, A.; Menon, S.K. Smart platform for the time since death determination from vitreous humor cystine. Biosensors Bioelectron. 2016, 86, 115-121,https://doi.org/10.1016/j.bios.2016.06.042.

16. Williams, T.; Soni, S.; White, J.; Can, G.; Javan, G.T. Evaluation of DNA degradation using flow cytometry: promising tool for postmortem interval determination. The American Journal of Forensic Medicine and Pathology 2015, 36, 104-110, https://doi.org/10.1097/PAF.0000000000000146.

17. Strasser, S., Zink, A., Kanda, G., Hinterdorfer, P., Peschel, O., Heckl, W.M., Nerlich, A.G. \& Thalhammer,S. Age determination of blood spots in forensic medicine by force spectroscopy.Int. J. Forensic. Sci. (2007), 170, 8-14, https://doi.org/10.1016/j.forsciint.2006.08.023.

18. Kumar, I.; Kumar, S.; Singh, M.; Kumari, K.; Kumar, D.; Ansari, K. Application of nanotechnology in forensic DNA and help to investigations on the crime scene analysis. World J Pharm Res 2016, 5, 266-276.

19. Castella, V.; Kummer, D.; Gehrig, C.; Hall, D. DNA extraction using the QIAsymphony: Evaluation of PCR inhibitor removal. Forensic Science International: Genetics Supplement Series 2011, 3, e69-e70, https://doi.org/10.1016/j.fsigss.2011.08.034.

20. McCord, B. Nanotechnology and its potential forensic DNA analysis Profiles in DNA. International forensic research institute 2006, 7-9. Available online: https://www.promega.in/resources/profiles-indna/2006/nanotechnology-and-itspotential- in-forensic-dnaanalysis/ (accessed on 05/04/2017).

21. Li, H.; Huang, J.; Lv, J.; An, H.; Zhang, X.; Zhang, Z.; Fan, C.; Hu, J. Nanoparticle PCR: Nanogold-Assisted PCR with Enhanced Specificity. Angew. Chem. 2005, 117, 5230-5233, https://doi.org/10.1002/ange.200500403.

22. Worley, C.G.; Wiltshire, S.S.; Miller, T.C.; Havrilla, G.J.; Majidi, V. Detection of visible and latent fingerprints using micro-X-ray fluorescence elemental imaging. J. Forensic Sci. 2006, 51, 57-63, https://doi.org/10.1111/j.1556-4029.2005.00006.x.

23. Arshad, A.; Farrukh, M.A.; Ali, S.; Khaleeq-ur-Rahman, M.; Tahir, M.A. Development of latent fingermarks on various surfaces using $\mathrm{ZnO}-\mathrm{SiO} 2$ nanopowder. J. Forensic Sci. 2015, 60, 1182-1187, https://doi.org/10.1111/1556-4029.12890.

24. Reynolds, A.J.; Jones, B.J.; Sears, V.; Bowman, V. Nano-scale analysis of titanium dioxide fingerprintdevelopment powders. Journal of Physics: Conference Series 2008, 126, 012069, https://doi.org/10.1088/1742-6596/126/1/012069.

25. Shilpa, C.J.; Basavaraj, R.B.; Darshan, G.P.; Premkumar, H.B.; Sharma, S.C.; Nagabhushana, H. New insights into the rapid deposition and visualization if latent fingerprints: Cyan light emitting $\mathrm{GdAlO}_{3}$ : $\mathrm{Ce} 3+$ nano fluorescent probe. Journal of Phytochemistry and Phytobiology A: Chemistry 2019, 376, 288-304, https://doi.org/10.1016/j.jphotochem.2019.02.027.

26. Han, M.H.Q.; Xing, B. Metallic Nanoparticle-Enabled Sensing of Drug-of-Abuse: An Attempt at Forensic Application. Application. Chemistry Europe. 2020, 21, 2512-2517. https://doi.org/10.1002/cbic.202000157.

27. Bhatt, P.V.; Pandey, G.; Tharmavaram, M.; Ratwani, D.; Hussain, C.M. Nanotechnology and Taggant Technology in Forensic Science. Technology in Forensic Science 2020, 14, https://doi.org/10.1002/9783527827688.ch14.

28. Ahmad, A.L.; Alawadhi, A.H.; Park, J.; Abdou, H.E.; Mohamed, A.A.; Evaluation of diazonium gold (III) salts in forensic chemistry: Latent fingerprint development on metal surfaces. Forensic Chemistry 2019, 13, 100144, https://doi.org/10.1016/j.forc.2019.100144.

29. Tharmavaram, M.; Pandey, G.; Hussain, C.M. Functionalized nanomaterial for forensic sample analysis. TrAC Trends in Analytical Chemistry 2019, 120, 115661, https://doi.org/10.1016/j.trac.2019.115661. 